\title{
The Douglas-Kroll-Heß Method: Convergence and Block-Diagonalization of Dirac Operators*
}

\author{
Heinz Siedentop and Edgardo Stockmeyer
}

\begin{abstract}
We show that the Douglas-Kroll block-diagonalization method for the Dirac operator with Coulomb potential is convergent in norm resolvent sense for coupling constant $\gamma$ less than $\gamma_{c}=0.37758$ which corresponds to atomic number 51 . Moreover, we give an explicit expression for the corresponding block-diagonalized Dirac operator.
\end{abstract}

\section{Introduction}

The one-particle Dirac operator is given by

$$
D_{\gamma}:=\boldsymbol{\alpha} \cdot \frac{1}{\mathrm{i}} \nabla+\beta+\gamma V
$$

acting in the Hilbert space $\mathfrak{H}:=L^{2}\left(\mathbb{R}^{3}\right) \otimes \mathbb{C}^{4}$. Here $\boldsymbol{\alpha}, \beta$ are the usual Dirac matrices. In this article we consider the Coulomb potential $V=-1 /|\cdot|$ with the coupling constant $\gamma \in(-1,1)$. (We use units in which the rationalized Planck constant, the mass of the particle, and the velocity of light are equal to one.) We choose $V$ as a Coulomb potential merely for convenience and definiteness. The method that we develop can be applied - in principle - to much more general situations. In fact it is not even necessary that the unperturbed operator is the free Dirac operator as the attentive reader will gather from the proofs.

It is well known that this operator is unbounded from below. Dirac postulated that negative energy states are already occupied by electrons, i.e., physical electrons are not allowed to exist in such states.

In most situations of chemical interest, pair-creation and excitations of the positronic degrees of freedom are far beyond the energy scale of the valence shell [30]. Therefore, it is a good approximation to neglect these quantum effects and "fill the Dirac sea". Mathematically this means that one should consider the Dirac operator projected onto its positive spectral subspace, i.e., $P(\gamma) D_{\gamma} P(\gamma)$ in the Hilbert space $P(\gamma) \mathfrak{H}$, where $P(\gamma):=\chi_{(0, \infty)}\left(D_{\gamma}\right)$ is the spectral projection of the Dirac operator $D_{\gamma}$ to its positive spectral subspace.

\footnotetext{
*This work was partially supported by the European Union's IHP network "Analysis \& Quantum" HPRN-CT-2002-00277 and the Volkswagen Stiftung through a cooperation grant. The authors thank Gheorghe Nenciu for pointing out reference [19]. Special thanks go to Hubert Kalf and Thomas Østergaard Sørensen for valuable discussions.
} 
For the free Dirac operator $D_{0}$ the corresponding projection $P_{0}:=P(0)$ is well known; in momentum space it is the matrix valued multiplication operator

$$
\mathcal{F} P_{0} \mathcal{F}^{-1}:=\frac{E(\mathfrak{p})+\boldsymbol{\alpha} \cdot \mathfrak{p}+\beta}{2 E(\mathfrak{p})}
$$

where $E(\mathfrak{p}):=\left(1+\mathfrak{p}^{2}\right)^{1 / 2}$ and $\mathcal{F}$ is the Fourier transform. In general, however, the corresponding projections are not explicitly known. Thus, it is important to develop an approximate procedure. One way of doing this was suggested in 1974 by M. Douglas and N.M. Kroll [7]. They start with the free case, i.e., $\gamma=0$, as the initial step. In the free case Foldy and Wouthuysen [9] showed that in momentum space the unitary transform

$$
\mathcal{F} U_{\mathrm{FW}} \mathcal{F}^{-1}:=\frac{1+\beta \boldsymbol{\alpha} \cdot \mathfrak{p}+E(\mathfrak{p})}{\sqrt{2 E(\mathfrak{p})(E(\mathfrak{p})+1)}} .
$$

maps the operator $D_{0}$ into a block-diagonal form, i.e.,

$$
\mathcal{F} U_{\mathrm{FW}} D_{0} U_{\mathrm{FW}}^{-1} \mathcal{F}^{-1}=\left(\begin{array}{cc}
E(\mathfrak{p}) & 0 \\
0 & -E(\mathfrak{p})
\end{array}\right) .
$$

For non-vanishing $\gamma$ the decoupling is done perturbatively. The method consists of a series of unitary transforms which are chosen to block-diagonalize the Hamiltonian up to a given order in the coupling constant $\gamma$. Every consecutive transform removes the lowest order in $\gamma$ of the remaining off-diagonal part. This allows the decoupling up to any power.

Brummelhuis et al. [5] observed that the first order term corresponds to the Brown-Ravenhall operator. It was extensively studied in the physics literature by Sucher and Hardekopf [27, 11, 12]. Evans et al. [8], Tix [28, 29], and Balinsky and Evans $[1,2]$ later showed some basic mathematical properties, e.g., positivity and self-adjointness.

Jansen and Heß [17] corrected an error in the derivation of the second order term by Douglas and Kroll. Later, Samzow et al. [25] extended the method to multi-particle operators. Actually, Heß et al. turned the method together with the efficient handling of the matrix elements of the corresponding two-component Hamiltonian into the "most successful two-components computational tool of the relativistic quantum chemistry" [3]. From a mathematical point of view the Jansen-Heß operator has been studied by Brummelhuis et al. [5, 26], by JakubaßaAmundsen [14, 16, 15], and by Jakubaßa-Amundsen and Iantchenko [13].

However, despite its success the question remains open as to whether proceeding to higher approximation really leads to better results as indicated numerically (Reiher and Wolf $[23,24]$ ). This paper endeavors to fill this gap: we will show that the spectrum of the approximate operators will become arbitrarily close to the spectrum of the Dirac operator as the approximation degree increases.

The organization of the paper is as follows: in Section 2 we review the Douglas-Kroll method. A unitary transform $U(\gamma)$, which block-diagonalizes the Dirac 
operator, is constructed in Section 3. In Section 4 we define the Douglas-Kroll operator of order $N$ and state our main results. In Section 5 , we prove that the operator $\left|D_{0}\right|^{1 / 2} U(\gamma)\left|D_{0}\right|^{-1 / 2}$ is analytic which implies that $\left|D_{0}\right|^{-1 / 2} U(\gamma) D_{\gamma} U(\gamma)^{-1}$ $\left|D_{0}\right|^{-1 / 2}$ is analytic as well. We use this in Section 6 to prove our main theorem. In Appendix A we state some useful equations. Finally, in Appendix B, we show that the expansion of the block-diagonalized Hamiltonian up to second order in powers of the coupling constant $\gamma$ indeed yields the Jansen-Heß operator. In addition, however, the expansion gives the possibility of obtaining further corrections to the energy. In fact, we can even guarantee that these contributions yield - when summed - the correct eigenvalue.

\section{The Douglas-Kroll method}

In order to be self-contained we shortly describe the Douglas-Kroll method (see [17] for details): the first step is to apply the (free) Foldy-Wouthuysen transform to $(1)$. We get

$$
U_{\mathrm{FW}} D_{\gamma} U_{\mathrm{FW}}^{-1}=: \beta E\left(\frac{1}{\mathrm{i}} \nabla\right)+\mathcal{E}_{1}+\mathcal{O}_{1}
$$

Here we decompose the difference of the Foldy-Wouthuysen transformed operator and $\beta E\left(\frac{1}{\mathrm{i}} \nabla\right)$ into its diagonal part $\mathcal{E}_{1}$ and off-diagonal part, i.e.,

$$
\begin{aligned}
\mathcal{E}_{1} & =\gamma\left(\beta_{+} U_{\mathrm{FW}} V U_{\mathrm{FW}}^{-1} \beta_{+}+\beta_{-} U_{\mathrm{FW}} V U_{\mathrm{FW}}^{-1} \beta_{-}\right), \\
\mathcal{O}_{1} & =\gamma \beta_{+} U_{\mathrm{FW}} V U_{\mathrm{FW}}^{-1} \beta_{-}+h . c . .
\end{aligned}
$$

Here $\beta_{ \pm}$are the orthogonal projection onto the upper respectively lower two components of four spinors, i.e., $\beta_{ \pm}=(1 \pm \beta) / 2$.

Note that both $\mathcal{E}_{1}$ and $\mathcal{O}_{1}$ are of order $\gamma$. The idea is then to remove the odd (off-diagonal) term to a higher order in $\gamma$. That can be carried out by a unitary transform

$$
U_{1}=\left(1+W_{1}^{2}\right)^{1 / 2}+W_{1},
$$

with $W_{1}^{*}=-W_{1}$. The operator $W_{1}$ is chosen such that - after applying $U_{1}$ and expanding in $\gamma$ - it removes the odd term proportional to $\gamma$ in the Hamiltonian. This will be the case, if $W_{1}$ satisfies the equation $\left[\beta E\left(\frac{1}{i} \nabla\right), W_{1}\right]=\mathcal{O}_{1}$. The resulting Hamiltonian can be written as

$$
\begin{aligned}
& U_{1} U_{\mathrm{FW}} D_{\gamma} U_{\mathrm{FW}}^{-1} U_{1}^{-1} \\
& \quad=\beta E\left(\frac{1}{\mathrm{i}} \nabla\right)+\mathcal{E}_{1}+\beta\left(W_{1} E\left(\frac{1}{\mathrm{i}} \nabla\right) W_{1}+\frac{1}{2}\left\{W_{1}^{2}, E\left(\frac{1}{\mathrm{i}} \nabla\right)\right\}\right)+\left[W_{1}, \mathcal{O}_{1}\right]+\rho_{\gamma}^{2}
\end{aligned}
$$

the term $\rho_{\gamma}^{2}$ represents operators which are $O\left(\gamma^{3}\right)$ on the diagonal and $O\left(\gamma^{2}\right)$ on the off-diagonal part. Those off-diagonal terms of second order in $\gamma$ can be removed using the method just described. This is the starting point of an iterative procedure which determines Hamiltonians that are formally diagonal up to any desired power in $\gamma$. We refer to the work of Wolf et al. [30] for details. 


\section{Exact block-diagonalization of the Dirac Hamiltonian}

The Dirac operator can be written in terms of its positive and negative spectral projections as

$$
D_{\gamma}=P(\gamma) D_{\gamma} P(\gamma)+P^{\perp}(\gamma) D_{\gamma} P^{\perp}(\gamma),
$$

here $P^{\perp}(\gamma):=1-P(\gamma)$. We note that to block-diagonalize $D_{\gamma}$ is equivalent to find a unitary transform $\tilde{U}(\gamma)$ such that

$$
\tilde{U}(\gamma) P(\gamma) \tilde{U}^{-1}(\gamma)=\beta_{+} .
$$

Since $U_{\mathrm{FW}} P(0) U_{\mathrm{FW}}^{*}=\beta_{+}$, it is enough to find a transform $U(\gamma)$ such that

$$
U(\gamma) P(\gamma) U^{-1}(\gamma)=P(0)
$$

Assuming the existence of such a transform $U(\gamma)$, we can say that $D_{\gamma}$ is unitarily equivalent to the operator

$$
\beta_{+} U_{\mathrm{FW}} U(\gamma) D_{\gamma} U^{-1}(\gamma) U_{\mathrm{FW}}^{-1} \beta_{+}+\beta_{-} U_{\mathrm{FW}} U(\gamma) D_{\gamma} U^{-1}(\gamma) U_{\mathrm{FW}}^{-1} \beta_{-}
$$

which is block-diagonal. Here the terms in the sum correspond to the electronic and positronic states respectively. Both terms can be viewed as acting on $L^{2}\left(\mathbb{R}^{3}\right) \otimes \mathbb{C}^{2}$ in a canonical way.

In the following we will construct such a unitary map $U(\gamma)$ and show its desired properties. We begin by recalling a property of the operator

$$
T_{\eta}:=|x|^{-1 / 2}\left(D_{0}-\mathrm{i} \eta\right)^{-1}|x|^{-1 / 2}
$$

with $\eta \in \mathbb{R}$ : Nenciu [22] conjectured and Kato [19], Formula 2.5, proved

$$
\left\|T_{\eta}\right\| \leq 1 \text {. }
$$

In the next lemmata we will show some basic properties of $P(\gamma)$.

Lemma 1 The projection $P(\gamma)$ has an analytic continuation into the disc $\mathcal{D}:=$ $\{\gamma \in \mathbb{C}|| \gamma \mid<1\}$.

Proof. Using the resolvent expansion given in [22], Lemma 2.2., we have for real $\gamma$ in the above disc

$$
\frac{1}{D_{\gamma}-\mathrm{i} \eta}=\frac{1}{D_{0}-\mathrm{i} \eta}+\gamma \frac{1}{D_{0}-\mathrm{i} \eta}|V|^{1 / 2} \frac{1}{1-\gamma T_{\eta}}|V|^{1 / 2} \frac{1}{D_{0}-\mathrm{i} \eta} .
$$

We note that the right-hand side is meaningful and norm-analytic in $\mathcal{D}$, since $\left\|\gamma T_{\eta}\right\|<1$ because of (12). This follows immediately by expanding $\left(1-\gamma T_{\eta}\right)^{-1}$.

To prove the analyticity of $P(\gamma)$ we use

$$
P(\gamma)=\frac{1}{2}+\frac{1}{2 \pi} \int_{-\infty}^{\infty} \frac{1}{D_{\gamma}-\mathrm{i} \eta} \mathrm{d} \eta
$$


where the integral is meant as Cauchy principal value in the strong sense (see Kato [18], Lemma VI-5.6). Therefore,

$$
\begin{aligned}
P(\gamma)-P(0) & =\frac{1}{2 \pi} \int_{-\infty}^{\infty}\left(D_{\gamma}-\mathrm{i} \eta\right)^{-1}-\left(D_{0}-\mathrm{i} \eta\right)^{-1} \mathrm{~d} \eta \\
& =\int_{-\infty}^{\infty} \sum_{n=0}^{\infty} \gamma^{n}\left(D_{0}-\mathrm{i} \eta\right)^{-1}|V|^{1 / 2}\left(T_{\eta}\right)^{n}|V|^{1 / 2}\left(D_{0}-\mathrm{i} \eta\right)^{-1} \mathrm{~d} \eta
\end{aligned}
$$

The integrand as function of $(n, \eta)$ is integrable in both the counting measure in $n$ and the Lebesgue measure in $\eta$. In fact,

$$
\begin{aligned}
& \left\|\gamma^{n}\left(D_{0}-\mathrm{i} \eta\right)^{-1}|V|^{1 / 2}\left(T_{\eta}\right)^{n}|V|^{1 / 2}\left(D_{0}-\mathrm{i} \eta\right)^{-1}\right\| \\
= & \gamma^{n} \sup _{f, g \in \mathfrak{H}} \frac{\left|\left(f,\left(D_{0}-\mathrm{i} \eta\right)^{-1}|V|^{1 / 2}\left(T_{\eta}\right)^{n}|V|^{1 / 2}\left(D_{0}-\mathrm{i} \eta\right)^{-1} g\right)\right|}{\|f\|\|g\|} \\
\leq & \frac{\pi}{2} \gamma^{n} \sup _{f, g \in \mathfrak{H}} \frac{\left\|\left.D_{0}\right|^{1 / 2}\left(D_{0}+\mathrm{i} \eta\right)^{-1} f\right\|}{\|f\|} \frac{\left\|\left|D_{0}\right|^{1 / 2}\left(D_{0}-\mathrm{i} \eta\right)^{-1} g\right\|}{\|g\|}
\end{aligned}
$$

where we used (12) followed by Kato's inequality $|\cdot|^{-1} \leq(\pi / 2) \sqrt{-\Delta}$. Furthermore, setting

$$
G_{\gamma}(\eta):=\left|D_{\gamma}\right|^{1 / 2}\left(D_{\gamma}-\mathrm{i} \eta\right)^{-1}
$$

we have

$$
\int_{-\infty}^{\infty}\left\|G_{\gamma}(\eta) \varphi\right\|^{2} \mathrm{~d} \eta=\pi\|\varphi\|^{2}
$$

for $\varphi \in \mathfrak{H}$ and $\gamma \in(-1,1)$. Therefore, using the Schwarz inequality

$$
\int_{-\infty}^{\infty} \mathrm{d} \eta\left\|G_{\gamma^{\prime}}(\eta) f\right\|\left\|G_{\gamma}(\eta) g\right\| \leq \pi\|f\|\|g\|
$$

for $\left|\gamma^{\prime}\right|<1$ and $f, g \in \mathfrak{H}$. Thus, the right-hand side of (16) is integrable, and the lemma is proved.

Lemma 2 For $\gamma \in(-\sqrt{3} / 2, \sqrt{3} / 2)$ we have the following estimate

$$
\|P(\gamma)-P(0)\| \leq \frac{\gamma^{2} \pi}{4} \nu_{\gamma}^{-1 / 2} .
$$

Here $\nu_{\gamma}$ is the constant defined in Appendix A. In particular, we have $\left\|P(\gamma)-P_{0}\right\|$ $<1$ for $|\gamma|<0.68418$.

Proof. Using the resolvent equation in (14), we write $P(\gamma)=P_{0}+\gamma P_{1}+Q_{\gamma}$ where $P_{0}=P(0), P_{1}:=\frac{1}{2 \pi} \int_{-\infty}^{\infty} \mathrm{d} \eta\left(D_{0}-\mathrm{i} \eta\right)^{-1} V\left(D_{0}-\mathrm{i} \eta\right)^{-1}$, and $Q_{\gamma}:=\frac{\gamma^{2}}{2 \pi} \int_{-\infty}^{\infty} \mathrm{d} \eta\left(D_{0}-\right.$ 
$\mathrm{i} \eta)^{-1} V\left(D_{0}-\mathrm{i} \eta\right)^{-1} V\left(D_{\gamma}-\mathrm{i} \eta\right)^{-1}$. Noting that $\left(P_{0}-P(\gamma)\right)^{2}=1-P_{0} P(\gamma) P_{0}-$ $P_{0}^{\perp} P(\gamma)^{\perp} P_{0}^{\perp}$ and also that $P_{0} P_{1} P_{0}=0=P_{0}^{\perp} P_{1} P_{0}^{\perp}$ we have

$$
\begin{aligned}
\left\|\left(P_{0}-P(\gamma)\right) \psi\right\|^{2} & =\left(P_{0}^{\perp} \psi, Q_{\gamma} P_{0}^{\perp} \psi\right)-\left(P_{0} \psi, Q_{\gamma} P_{0} \psi\right) \\
& \leq\left\|Q_{\gamma}\right\|\left(\left\|P_{0}^{\perp} \psi\right\|^{2}+\left\|P_{0} \psi\right\|^{2}\right)=\left\|Q_{\gamma}\right\|\|\psi\|^{2} .
\end{aligned}
$$

To compute the norm of $Q_{\gamma}$ we estimate for $f, g \in \mathfrak{H}$

$$
\begin{aligned}
\mid\left(f,\left(D_{0}-\mathrm{i} \eta\right)^{-1}\right. & \left.V\left(D_{0}-\mathrm{i} \eta\right)^{-1} V\left(D_{\gamma}-\mathrm{i} \eta\right)^{-1} g\right) \mid \\
& \leq\left\|T_{-\eta}|V|^{1 / 2}\left(D_{0}+\mathrm{i} \eta\right)^{-1} f\right\|\left\||V|^{1 / 2}\left(D_{\gamma}-\mathrm{i} \eta\right)^{-1} g\right\| \\
& \leq \frac{\pi}{2}\left\|\left|D_{0}\right|^{1 / 2}\left(D_{0}+\mathrm{i} \eta\right)^{-1} f\right\|\left\|\left|D_{0}\right|^{1 / 2}\left(D_{\gamma}-\mathrm{i} \eta\right)^{-1} g\right\| \\
& \leq \nu_{\gamma}^{-1 / 2} \frac{\pi}{2}\left\|G_{0}(\eta) f\right\|\left\|\mid G_{\gamma}(\eta) g\right\|
\end{aligned}
$$

where we have used (12), Kato's inequality, and (43). The lemma follows now from (19).

Theorem 1 For $|\gamma|<0.68418$

$$
U(\gamma)=\left(P_{0} P(\gamma)+P_{0}^{\perp} P^{\perp}(\gamma)\right)\left(1-\left(P_{0}-P(\gamma)\right)^{2}\right)^{-1 / 2}
$$

is analytic in $\gamma$ and unitary. Moreover, it fulfills $U(\gamma) P(\gamma) U^{*}(\gamma)=P_{0}$.

Proof. The operator is well defined due to Lemma 2. The unitarity as well as the Property (9) can be easily verified using that $\left(P_{0}-P(\gamma)\right)^{2}$ is equal to $1-$ $\left(P_{0} P(\gamma) P_{0}+P_{0}^{\perp} P(\gamma)^{\perp} P_{0}^{\perp}\right)$ and commutes with $P_{0}$ and $P(\gamma)$. Finally, the analyticity follows from Lemma 1 and the fact that $(1-A)^{-1 / 2}$ has a convergent expansion in powers of $A$ as long as $\|A\|<1$.

First, we note that the above strategy of diagonalization can be found in [18] but has not been applied to Dirac operators in this context. The block diagonalization of such operators under the condition that the potential is bounded by 1 has been carried out by Langer and Tretter [20] by different means.

Next, we remark that the transform $U(\gamma)$ is not unique: Already Kato commented [18], II-4.2, that there are other ways to derive such transforms which allow to relax the condition on $\gamma$. However, they have the disadvantage that the expressions are recursively determined instead of being given in a closed algebraic form. Nevertheless, all of these transforms coincide up to order $\gamma^{2}$ (inclusive). This feature was also used in [30], where the authors explore the fact that the ansatz in (5) is not unique. For another expansion method see [16].

\section{Definition of the Douglas-Kroll operator}

The fully block-diagonalized Dirac operator is

$$
H_{\gamma}:=\beta_{+} U_{\mathrm{FW}} U(\gamma) D_{\gamma} U^{-1}(\gamma) U_{\mathrm{FW}}^{-1} \beta_{+}+\beta_{-} U_{\mathrm{FW}} U(\gamma) D_{\gamma} U^{-1}(\gamma) U_{\mathrm{FW}}^{-1} \beta_{-} .
$$


In order to make contact with the Douglas-Kroll method we consider the expansion in $\gamma$ of $U(\gamma) D_{\gamma} U^{-1}(\gamma)$ obtaining the formal operator

$$
\sum_{j=0}^{N} \gamma^{j} \sum_{k=0}^{j} u_{k} D_{0} u_{j-k}^{*}+\sum_{j=0}^{N-1} \gamma^{j+1} \sum_{k=0}^{j} u_{k} V u_{j-k}^{*}
$$

Here $u_{j}$, for $j \in \mathbb{N}$, are the coefficients of the expansion of $U(\gamma)$, i.e.,

$$
U(\gamma)=\sum_{j=0}^{\infty} \gamma^{j} u_{j}
$$

Let us define the operator

$$
R_{\gamma}^{N}:=U(\gamma) D_{\gamma} U(\gamma)^{-1}-\sum_{j=0}^{N} \gamma^{j} \sum_{k=0}^{j} u_{k} D_{0} u_{j-k}^{*}-\sum_{j=0}^{N-1} \gamma^{j+1} \sum_{k=0}^{j} u_{k} V u_{j-k}^{*}
$$

acting in $C_{0}^{\infty}\left(\mathbb{R}^{3}\right)^{4}$. We can now formulate one of our main results.

Theorem 2 If $\gamma$ or $1 / N$ are small enough, the operator $(25)$, i.e., $U(\gamma) D_{\gamma} U(\gamma)^{-1}-$ $R_{\gamma}^{N}$, admits a distinguished self-adjoint extension, the pseudo-Friedrichs extension $d_{\gamma}^{N}$ for which $D\left(d_{\gamma}^{N}\right) \subset D\left(\left|D_{0}\right|^{1 / 2}\right) \equiv H^{1 / 2}\left(\mathbb{R}^{3}\right)^{4}$. Moreover, for $|\gamma|<\gamma_{c}$ with $\gamma_{c}=$ 0.37758 , the operator $d_{\gamma}^{N}$ converges in norm resolvent sense to $U(\gamma) D_{\gamma} U(\gamma)^{-1}$.

We are not yet in a position to give the proof of this result. We postpone it to Section 6 and define instead the $N$-th order Douglas-Kroll operator as

$$
H_{\gamma}^{N}:=\beta_{+} U_{\mathrm{FW}} d_{\gamma}^{N} U_{\mathrm{FW}}^{-1} \beta_{+}+\beta_{-} U_{\mathrm{FW}} d_{\gamma}^{N} U_{\mathrm{FW}}^{-1} \beta_{-} .
$$

One can explicitly verify that the first terms correspond precisely to the ones obtained in [17] (see Appendix B). The important fact following from Theorem 2 is, that the eigenvalues of $d_{\gamma}^{N}$ converge to the eigenvalues of $D_{\gamma}$ as the degree $N$ of the approximation tends to infinity:

Corollary 1 For $\gamma<\gamma_{c}$ hold: If $\lambda \in \mathbb{R} \backslash \sigma\left(D_{\gamma}\right)$, then $\lambda \notin \sigma\left(d_{\gamma}^{N}\right)$ for all large enough $N$. If $\lambda \in \sigma\left(D_{\gamma}\right)$, then there exists $\lambda_{N} \in \sigma\left(d_{\gamma}^{N}\right)$ for which $\lim _{N \rightarrow \infty} \lambda_{N}=\lambda$.

For a proof of this result see, e.g., [6] Chapter 2.

\section{$5 \quad$ Analyticity of $\left|D_{0}\right|^{1 / 2} U(\gamma)\left|D_{0}\right|^{-1 / 2}$}

We first need some technical results:

Lemma 3 The operator $\left|D_{0}\right|^{1 / 2}\left(P(\gamma)-P_{0}\right)\left|D_{0}\right|^{-1 / 2}$ has an analytic continuation into the disc $\mathcal{D}:=\{\gamma \in \mathbb{C}|| \gamma \mid<1 / 2\}$. 
Proof. We follow the same strategy as in Lemma 1 . The analyticity follows from (15), the fact that $|V|^{1 / 2} T_{\eta}^{n}=\left(|V|\left(D_{0}-\mathrm{i} \eta\right)^{-1}\right)^{n}|V|^{1 / 2}$, and the following estimate

$$
\begin{aligned}
& \left\|\gamma^{n}\left|D_{0}\right|^{1 / 2}\left(D_{0}-\mathrm{i} \eta\right)^{-1}\left(|V|\left(D_{0}-\mathrm{i} \eta\right)^{-1}\right)^{n}|V|\left|D_{0}\right|^{-1 / 2}\left(D_{0}-\mathrm{i} \eta\right)^{-1}\right\| \\
\leq & 2(2 \gamma)^{n} \sup _{f, g \in \mathfrak{H}}\left\|G_{0}(\eta) f\right\|\left\|G_{0}(\eta) g\right\|
\end{aligned}
$$

where we used Hardy's inequality $|\cdot|^{-2} \leq 4\left|D_{0}\right|^{2}$.

Lemma 4 For $\gamma \in(-\sqrt{3} / 2, \sqrt{3} / 2)$ we have the following estimate

$$
||\left|D_{0}\right|^{1 / 2}\left(P(\gamma)-P_{0}\right)\left|D_{0}\right|^{-1 / 2} \| \leq \gamma \nu_{\gamma}^{-1} \sqrt{1+\frac{\pi}{2} \gamma} .
$$

In particular, we have $\left\|\left|D_{0}\right|^{1 / 2}\left(P(\gamma)-P_{0}\right)\left|D_{0}\right|^{-1 / 2}\right\|<1$ for $|\gamma|<\gamma_{c}$.

Proof. For $f, g \in H^{1 / 2}\left(\mathbb{R}^{3}\right)^{4}$ we have

$$
\begin{aligned}
& \left|\left(f,\left|D_{0}\right|^{1 / 2}\left(P(\gamma)-P_{0}\right)\left|D_{0}\right|^{-1 / 2} g\right)\right| \\
= & \frac{\gamma}{2 \pi}\left|\int_{-\infty}^{\infty}\left(f,\left|D_{0}\right|^{1 / 2}\left(D_{0}-\mathrm{i} \eta\right)^{-1} V\left(D_{\gamma}-\mathrm{i} \eta\right)^{-1}\left|D_{0}\right|^{-1 / 2} g\right) \mathrm{d} \eta\right| \\
\leq & \frac{\gamma}{2 \pi} \int_{-\infty}^{\infty}\left\|\left|D_{0}\right|^{1 / 2}\left(D_{0}+\mathrm{i} \eta\right)^{-1} f\right\|\left\||V|\left(D_{\gamma}-\mathrm{i} \eta\right)^{-1}\left|D_{0}\right|^{-1 / 2} g\right\| \mathrm{d} \eta .
\end{aligned}
$$

Now, using Hardy's inequality and (43) we estimate

$$
\begin{aligned}
\left\||V|\left(D_{\gamma}-\mathrm{i} \eta\right)^{-1}\left|D_{0}\right|^{-1 / 2} g\right\| & \leq 2\left\|\left|D_{0}\right|\left(D_{\gamma}-\mathrm{i} \eta\right)^{-1}\left|D_{0}\right|^{-1 / 2} g\right\| \\
& \leq 2 \nu_{\gamma}^{-1}\left\|\left|D_{\gamma}\right|\left(D_{\gamma}-\mathrm{i} \eta\right)^{-1}\left|D_{0}\right|^{-1 / 2} g\right\| .
\end{aligned}
$$

Therefore, (19) implies

$$
\left|\left(f,\left|D_{0}\right|^{1 / 2}\left(P(\gamma)-P_{0}\right)\left|D_{0}\right|^{-1 / 2} g\right)\right| \leq \gamma \nu_{\gamma}^{-1}\|f\|\left\|\left.|| D_{\gamma}\right|^{1 / 2}\left|D_{0}\right|^{-1 / 2} g\right\|
$$

which leads to the desired result after using Kato's inequality on the right-hand side of the latter expression.

Lemma 5 The operator $\left|D_{0}\right|^{1 / 2} U(\gamma)\left|D_{0}\right|^{-1 / 2}$ is bounded and analytic in $\gamma$ for $|\gamma|$ $<\gamma_{c}$.

Proof. Using properties of orthogonal projections we have the operator identity $P_{0} P(\gamma)+P_{0}^{\perp} P(\gamma)^{\perp}=1-\left(P_{0}^{\perp}-P_{0}\right)\left(P(\gamma)-P_{0}\right)$. Inserting the latter equation in (23) we write

$$
U(\gamma)=\left(1-\left(P_{0}^{\perp}-P_{0}\right)\left(P(\gamma)-P_{0}\right)\right)\left(1-\left(P_{0}-P(\gamma)\right)^{2}\right)^{-1 / 2} .
$$


Let us note first that

$$
\begin{aligned}
\left|D_{0}\right|^{1 / 2}\left(1-\left(P_{0}^{\perp}-P_{0}\right)(P(\gamma)\right. & \left.\left.-P_{0}\right)\right)\left|D_{0}\right|^{-1 / 2} \\
& =1-\left(P_{0}^{\perp}-P_{0}\right)\left|D_{0}\right|^{1 / 2}\left(P(\gamma)-P_{0}\right)\left|D_{0}\right|^{-1 / 2}
\end{aligned}
$$

is bounded by Lemma 4 . Secondly we use (44) and look at

$$
\begin{aligned}
&\left|D_{0}\right|^{1 / 2}(1-\left.\left(P_{0}-P(\gamma)\right)^{2}\right)^{-1 / 2}\left|D_{0}\right|^{-1 / 2} \\
&=\frac{1}{\pi} \int_{-1}^{1} \frac{1}{\sqrt{1-y^{2}}}\left|D_{0}\right|^{1 / 2} \frac{1}{\left(1-y\left(P_{0}-P(\gamma)\right)\right)}\left|D_{0}\right|^{-1 / 2} \mathrm{~d} y \\
& \quad=\frac{1}{\pi} \int_{-1}^{1} \frac{1}{\sqrt{1-y^{2}}} \frac{1}{\left(1-y\left|D_{0}\right|^{1 / 2}\left(P_{0}-P(\gamma)\right)\left|D_{0}\right|^{-1 / 2}\right)} \mathrm{d} y
\end{aligned}
$$

By Lemma 4 we can estimate the operator inside the integral above as

$$
\begin{aligned}
\left(1-y\left|D_{0}\right|^{1 / 2}\left(P_{0}-P(\gamma)\right)\left|D_{0}\right|^{-1 / 2}\right)^{-1} & \\
& \leq\left[1-|y|\left\|\left|D_{0}\right|^{1 / 2}\left(P_{0}-P(\gamma)\right)\left|D_{0}\right|^{-1 / 2}\right\|\right]^{-1} .
\end{aligned}
$$

Therefore,

$$
\begin{aligned}
\left\|\left|D_{0}\right|^{1 / 2}\left(1-\left(P_{0}-P(\gamma)\right)^{2}\right)^{-1 / 2}\left|D_{0}\right|^{-1 / 2}\right\| & \\
& \leq\left[1-\left\|\left|D_{0}\right|^{1 / 2}\left(P_{0}-P(\gamma)\right)\left|D_{0}\right|^{-1 / 2}\right\|\right]^{-1}
\end{aligned}
$$

which together with Lemmata 3 and 4 completes the proof.

\section{Convergence of the Douglas-Kroll Hamiltonian}

Lemma 6 For $\gamma<\gamma_{c}$, the operator $\left|D_{0}\right|^{-1 / 2} U(\gamma) D_{\gamma} U(\gamma)^{-1}\left|D_{0}\right|^{-1 / 2}$ is bounded and analytic in $\mathfrak{H}$.

Proof. We write

$$
\begin{aligned}
\left|D_{0}\right|^{-1 / 2} U(\gamma) D_{\gamma} U(\gamma)^{-1} & \left|D_{0}\right|^{-1 / 2}=\left(\left|D_{0}\right|^{-1 / 2} U(\gamma)\left|D_{0}\right|^{1 / 2}\right) \\
& \times\left(\left|D_{0}\right|^{-1 / 2} D_{\gamma}\left|D_{0}\right|^{-1 / 2}\right)\left(\left|D_{0}\right|^{1 / 2} U(\gamma)^{-1}\left|D_{0}\right|^{-1 / 2}\right)
\end{aligned}
$$

and observe that each term is bounded and analytic by Lemma 5 in one case or by Kato's inequality in the other.

An immediate consequence of Lemma 6 is that, for $\gamma<\gamma_{c}$

$$
\left\|\left|D_{0}\right|^{-1 / 2} R_{\gamma}^{N}\left|D_{0}\right|^{-1 / 2}\right\| \rightarrow 0
$$

as $N \rightarrow \infty$. 
Proof of Theorem 2. Note first that for $u \in C_{0}^{\infty}\left(\mathbb{R}^{3}\right)^{4}$ the following estimate holds

$$
\begin{aligned}
& \left|\left(u,\left|D_{\gamma}\right|^{-\frac{1}{2}} U^{-1}(\gamma) R_{\gamma}^{N} U(\gamma)\left|D_{\gamma}\right|^{-\frac{1}{2}} u\right)\right| \\
& \leq\left\|\left|D_{0}\right|^{-\frac{1}{2}} R_{\gamma}^{N}\left|D_{0}\right|^{-\frac{1}{2}}\right\|\left\|\left|D_{0}\right|^{\frac{1}{2}} U(\gamma)\left|D_{0}\right|^{-\frac{1}{2}}\right\|^{2}\left\|\left|D_{\gamma}\right|^{-\frac{1}{2}}\left|D_{0}\right|\left|D_{\gamma}\right|^{-\frac{1}{2}}\right\|\|u\|^{2} .
\end{aligned}
$$

The left-hand side is obviously bounded for $\gamma<\gamma_{c}$. We proceed now to prove the self-adjointness of $d_{\gamma}^{N}$. We take the Pseudo-Friedrichs extension (see [18] Theorem VI-3.11) of this operator. The operator $U(\gamma)$ leaves the Sobolev space $H^{1 / 2}\left(\mathbb{R}^{3}\right)^{4}$ invariant by Lemma 5 . Thus it is enough to prove

$$
\left|\left(u, U^{-1}(\gamma) R_{\gamma}^{N} U(\gamma) u\right)\right| \leq b_{\gamma, N}\left(u,\left|D_{\gamma}\right| u\right) \quad u \in C_{0}^{\infty}\left(\mathbb{R}^{3}\right)^{4}
$$

with $b_{\gamma, N}<1$. This, however, is clearly true because (41), if $\gamma$ is small or $N$ is big.

Finally, the resolvent convergence of $d_{\gamma}^{N}$ to $U(\gamma) D_{\gamma} U^{-1}(\gamma)$ follows from Corollary VI-3.12 in [18] and (40).

\section{A Some useful equations}

We introduce the constant $\nu_{\gamma}^{2}:=(1 / 2)\left\{1+C_{\gamma}^{2}-\left[\left(1-C_{\gamma}^{2}\right)^{2}+4 \gamma^{2} C_{\gamma}^{2}\right]^{1 / 2}\right\}$ with $C_{\gamma}:=(1 / 3)\left[\left(4 \gamma^{2}+9\right)^{1 / 2}-4 \gamma\right]$. Then

Lemma 7 (Morozov [21]) For $\gamma \in(-\sqrt{3} / 2, \sqrt{3} / 2)$ we have

$$
\left|D_{\gamma}\right|^{2} \geq \nu_{\gamma}^{2}\left|D_{0}\right|^{2}
$$

We note that this result generalizes [4], Inequality (2) to the massive case.

The following formula is useful for an integral representation of the unitary operator $U(\gamma)$. For $|a|<1$ we have

$$
\left(1-a^{2}\right)^{-1 / 2}=\frac{1}{\pi} \int_{-1}^{1} \frac{1}{\sqrt{1-y^{2}}} \frac{1}{(1-y a)} \mathrm{d} y .
$$

In order to verify the above see, e.g., [10] Formula 3.197.4.

\section{B Derivation of the Hamiltonian}

In this section we explicitly derive the block-diagonalized Hamiltonians up to the order $N=2$. In particular, we will look at (28) and compare it with (6). Expanding the projection $P(\gamma)$ yields for $\gamma<1$

$$
P(\gamma)=\sum_{n=0}^{\infty} \gamma^{n} P_{n}
$$


with

$$
P_{n}=\frac{1}{2 \pi} \int_{-\infty}^{\infty}\left(\frac{-1}{D_{0}-\mathrm{i} \eta} V\right)^{n} \frac{1}{D_{0}-\mathrm{i} \eta} \mathrm{d} \eta .
$$

Next, we compute the first coefficients of the expansion of $U(\gamma)$ as given by (23) and (26) and get

$$
\begin{aligned}
& u_{0}=1, \\
& u_{1}=\left(P_{0}-P_{0}^{\perp}\right) P_{1}, \\
& u_{2}=\left(P_{0}-P_{0}^{\perp}\right) P_{2}+\frac{1}{2} P_{1}^{2},
\end{aligned}
$$

here $P_{i}$ with $i \in \mathbb{N}$ are given in (46). The following properties for the $P_{i}$ stem from the fact that $P(\gamma)$ is idempotent, and will be used later:

$$
\begin{aligned}
& P_{1}=P_{0} P_{1}+P_{1} P_{0}, \\
& P_{2}=P_{2} P_{1}+P_{2} P_{0}+P_{1}^{2} .
\end{aligned}
$$

For $N=0$ we have $d_{\gamma}^{0}=D_{0}$. Therefore,

$$
H_{\gamma}^{0}=U_{\mathrm{FW}} P_{0} D_{0} P_{0} U_{\mathrm{FW}}^{-1}+U_{\mathrm{FW}} P_{0}^{\perp} D_{0} P_{0}^{\perp} U_{\mathrm{FW}}^{-1}=\beta \sqrt{-\Delta+m},
$$

which corresponds to the first term in (6). In the above we used

$$
\beta_{+} U_{\mathrm{FW}}=U_{\mathrm{FW}} P_{0}
$$

For $N=1$ we have

$$
d_{\gamma}^{1}=d_{\gamma}^{0}+\gamma D_{0} u_{1}^{*}+\gamma u_{1} D_{0}+\gamma V
$$

the terms $u_{1} D_{0}$ and its adjoint do not contribute to $H_{\gamma}^{1}$. In fact, using that (51) in (28), and the fact that since (48) holds, $P_{0} P_{1} P_{0}=P_{0}^{\perp} P_{1} P_{0}^{\perp}=0$ we can eliminate those terms. Therefore,

$$
H_{\gamma}^{1}=U_{\mathrm{FW}} P_{0}\left(D_{0}+\gamma V\right) P_{0} U_{\mathrm{FW}}^{-1}+U_{\mathrm{FW}} P_{0}^{\perp}\left(D_{0}+\gamma V\right) P_{0}^{\perp} U_{\mathrm{FW}}^{-1},
$$

which corresponds to the first two terms in (6). The electronic part is the BrownRavenhall operator. Note that $\mathcal{E}$ in (6) fulfills $U_{\mathrm{FW}}^{-1} \mathcal{E} U_{\mathrm{FW}}=P_{0} \gamma V P_{0}+P_{0}^{\perp} \gamma V P_{0}^{\perp}$.

Finally, we consider the case $N=2$, we have

$$
d_{\gamma}^{2}=d_{\gamma}^{1}+\gamma^{2}\left(D_{0} u_{2}^{*}+u_{2} D_{0}+u_{1} D_{0} u_{1}^{*}+u_{1} V+V u_{1}^{*}\right)
$$

We note that

$$
\begin{aligned}
P_{0} u_{2} P_{0} & =P_{0}\left(P_{0} P_{2} P_{0}+\frac{1}{2} P_{1}^{2}\right) P_{0}=P_{0}\left(-\frac{1}{2} P_{1}^{2}\right) P_{0}, \\
P_{0}^{\perp} u_{2} P_{0}^{\perp} & =P_{0}^{\perp}\left(-\frac{1}{2} P_{1}^{2}\right) P_{0}^{\perp}
\end{aligned}
$$


where we used (49) projected by $P_{0}$ and $P_{0}^{\perp}$, respectively. Therefore,

$$
\begin{aligned}
H_{\gamma}^{2}= & H_{\gamma}^{1}+U_{\mathrm{FW}} P_{0}\left(P_{1} D_{0} P_{1}-\left\{\frac{1}{2} P_{1}^{2}, D_{0}\right\}+P_{1} V+V P_{1}\right) P_{0} U_{\mathrm{FW}}^{-1} \\
& +U_{\mathrm{FW}} P_{0}^{\perp}\left(P_{1} D_{0} P_{1}-\left\{\frac{1}{2} P_{1}^{2}, D_{0}\right\}-P_{1} V-V P_{1}\right) P_{0}^{\perp} U_{\mathrm{FW}}^{-1},
\end{aligned}
$$

which are the terms that appear in (6), since

$$
\begin{aligned}
U_{\mathrm{FW}}^{-1} W_{1} U_{\mathrm{FW}} & =\left(P_{0}-P_{0}^{\perp}\right) P_{1}, \\
U_{\mathrm{FW}}^{-1} \mathcal{O}_{1} U_{\mathrm{FW}} & =P_{0} \gamma V P_{0}^{\perp}+P_{0}^{\perp} \gamma V P_{0} .
\end{aligned}
$$

Here the electronic part of $H_{\gamma}^{2}$ is the Jansen-Heß operator.

\section{References}

[1] A.A. Balinsky and W.D. Evans, On the virial theorem for the relativistic operator of Brown and Ravenhall, and the absence of embedded eigenvalues, Lett. Math. Phys. 44(3), 233-248 (1998).

[2] A.A. Balinsky and W.D. Evans, Stability of one-electron molecules in the Brown-Ravenhall model, Comm. Math. Phys. 202(2), 481-500 (1999).

[3] Maria Barysz and Andrzej Sadlej, Two-component methods of relativistic quantum chemistry: From the Douglas-Kroll approximation to the exact twocomponent formalism, J. Molec. Struc. (Theochem) 573, 181-200 (2001).

[4] Raymond Brummelhuis, Norbert Röhrl, and Heinz Siedentop, Stability of the relativistic electron-positron field of atoms in Hartree-Fock approximation: Heavy elements, Doc. Math., J. DMV, 6, 1-8 (2001).

[5] Raymond Brummelhuis, Edgardo Stockmeyer, and Heinz Siedentop, The ground state energy of relativistic one-electron atoms, Doc. Math., J. DMV 7, 167-182 (2002).

[6] E.B. Davies, Spectral theory and differential operators, Cambridge Studies in Advanced Mathematics. 42. Cambridge: Cambridge Univ. Press. ix, 182 p., 1996.

[7] Marvin Douglas and Norman M. Kroll, Quantum electrodynamical corrections to the fine structure of helium, Annals of Physics 82, 89-155 (1974).

[8] William Desmond Evans, Peter Perry, and Heinz Siedentop, The spectrum of relativistic one-electron atoms according to Bethe and Salpeter, Comm. Math. Phys. 178 (3), 733-746 (1996). 
[9] Leslie L. Foldy and Siegfried A. Wouthuysen, On the Dirac Theory of Spin 1/2 Particles and its Non-Relativistic Limit, Phys. Rev., II. Ser. 78 29-36 (1950).

[10] I.S. Gradshteyn and I.M. Ryzhik, Table of Integrals, Series, and Products, Academic Press, 4th edition, 1980.

[11] G. Hardekopf and J. Sucher, Relativistic wave equations in momentum space, Phys. Rev. A 30 (2), 703-711 (1984).

[12] G. Hardekopf and J. Sucher, Critical coupling constants for relativistic wave equations and vacuum breakdown in quantum electrodynamics, Phys. Rev. A 31 (4), 2020-2029 (1985).

[13] A. Iantchenko and D.H. Jakubaßa-Amundsen, On the positivity of the JansenHeß operator for arbitrary mass, Ann. Henri Poincaré 4 (6), 1083-1099 (2003).

[14] D.H. Jakubaßa-Amundsen, The essential spectrum of relativistic one-electron ions in the Jansen-Hess model, Math. Phys. Electron. J. 8, Paper 3, 30 pp. (electronic) (2002).

[15] D.H. Jakubassa-Amundsen, Pseudorelativistic operator for a two-particle ion. Physical Review A $\mathbf{7 1}$ (2005).

[16] Doris Jakubaßa-Amundsen, Spectral Theory of the Atomic Dirac Operator in the No-Pair Formalism, PhD thesis, Fakultät für Mathematik, Informatik und Statistik; Ludwig-Maximilians-Universität München, 2004.

[17] Georg Jansen and Bernd A. Heß, Revision of the Douglas-Kroll transformation. Physical Review A 39 (11), 6016-6017 (1989).

[18] Tosio Kato, Perturbation Theory for Linear Operators, volume 132 of Grundlehren der mathematischen Wissenschaften, Springer-Verlag, Berlin, 1 edition, 1966.

[19] Tosio Kato, Holomorphic families of Dirac operators, Math. Z. 183, 399-406 (1983).

[20] Heinz Langer and Christiane Tretter, Diagonalization of certain block operator matrices and applications to Dirac operators, In Bart, H. (ed.) et al., Operator theory and analysis. The M. A. Kaashoek anniversary volume. Proceedings of the workshop, Amsterdam, Netherlands, November 12-14, $199 \%$. Basel: Birkhäuser. Oper. Theory, Adv. Appl. 122, 331-358 (2001).

[21] Sergey Morozov, Extension of a minimax principle for Coulomb-Dirac operators, Master's thesis, Mathematisches Institut, Ludwig-Maximilians-Universität, Theresienstr. 39, 80333 München, Germany, August 2004. 
[22] G. Nenciu, Self-adjointness and invariance of the essential spectrum for Dirac operators defined as quadratic forms, Comm. Math. Phys. 48, 235-247 (1976).

[23] Markus Reiher and Alexander Wolf, Exact decoupling of the Dirac Hamiltonian. I. General theory, J. Chem. Phys. 121 (5), 2037-2047 (2004).

[24] Markus Reiher and Alexander Wolf, Exact decoupling of the Dirac Hamiltonian. II. The generalized Douglas-Kroll-Hess transformation up to arbitrary order, J. Chem. Phys. 121 (22), 10945-10956 (2004).

[25] Reinhardt Samzow, Bernd A. Hess, and Georg Jansen, The 2-electron terms of the no-pair Hamiltonian, J. Chem. Phys 96 (2), 1227-1231 (1992).

[26] Edgardo Stockmeyer, Electrones Relativistas en un Contexto de la Estabilidad de la Materia, PhD thesis, Facultad de Física de la Pontificia Universidad Católica de Chile, Santiago, Chile, May 2002.

[27] J. Sucher, Foundations of the relativistic theory of many-electron atoms, Phys. Rev. A 22 (2), 348-362 (1980).

[28] C. Tix, Lower bound for the ground state energy of the no-pair Hamiltonian, Phys. Lett. B 405 (3-4), 293-296 (1997).

[29] C. Tix, Self-adjointness and spectral properties of a pseudo-relativistic Hamiltonian due to Brown and Ravenhall, Preprint, mp-arc: 97-441, 1997.

[30] Alexander Wolf, Markus Reiher, and Bernd Hess, The generalized DouglasKroll transformation, J. Chem. Phys. 117 (20), 9215-9226 (2002).

Heinz Siedentop and Edgardo Stockmeyer

Mathematisches Institut

Ludwig-Maximilians-Universität München

Theresienstraße 39

D-80333 München

Germany

email: h.s@lmu.de

email: stock@math.lmu.de

Communicated by Vincent Rivasseau

submitted 26/02/05, accepted 12/04/05

\section{(17) To access this journal online: (2P) http://www.birkhauser.ch}

